\title{
Existence and multiplicity results for a class of fractional differential inclusions with boundary conditions
}

\author{
Peng Zhang and Yanping Gong*
}

*Correspondence:

penggreatpaper@126.com Business School, Central South University, Changsha, Hunan 410083, P.R. China

\begin{abstract}
In this paper, we study the existence and multiplicity results of solutions for some class of fractional differential inclusions with boundary conditions. Some existence and multiplicity results of solutions are given by using the least action principle and minmax methods in nonsmooth critical point theory. Recent results in the literature are generalized and improved. Some examples are given in the paper to illustrate our main results.
\end{abstract}

MSC: $26 \mathrm{~A} 33 ; 26 \mathrm{~A} 42 ; 58 \mathrm{E} 05 ; 70 \mathrm{H} 05$

Keywords: fractional differential inclusions; nonsmooth critical point theory; boundary value problem; variational methods

\section{Introduction}

In this paper, we consider the fractional boundary value problem (BVP for short) for the following differential inclusion:

$$
\left\{\begin{array}{l}
-\frac{d}{d t}\left(\frac{1}{2} 0 D_{t}^{-\beta}\left(u^{\prime}(t)\right)+\frac{1}{2}{ }_{t} D_{T}^{-\beta}\left(u^{\prime}(t)\right)\right) \in \partial F(t, u(t)), \quad \text { a.e. } t \in[0, T], \\
u(0)=u(T)=0,
\end{array}\right.
$$

where $T>0,{ }_{0} D_{t}^{-\beta}$ and ${ }_{t} D_{T}^{-\beta}$ are the left and right Riemann-Liouville fractional integrals of order $0 \leq \beta<1$ respectively, $F:[0, T] \times \mathbb{R}^{N} \rightarrow \mathbb{R}$ satisfies the following assumptions:

(A) $F(t, x)$ is measurable in $t$ for every $x \in \mathbb{R}^{N}$ and locally Lipschitz in $x$ for a.e. $t \in[0, T]$, $F(t, 0) \in L^{1}(0, T)$ and there exist $f, g \in L^{\infty}\left(0, T ; \mathbb{R}^{+}\right)$and $v \in[0, \infty)$ such that

$$
\zeta \in \partial F(t, x) \quad \Rightarrow \quad|\zeta| \leq f(t)|x|^{\nu}+g(t)
$$

for a.e. $t \in[0, T]$ and all $x \in \mathbb{R}^{N}$.

Differential equations with fractional order are generalization of ordinary differential equations to non-integer order. Fractional differential equations have received increasing attention during recent years, since the behavior of physical systems can be properly described by using fractional order system theory. So fractional differential equations got the attention of many researchers and considerable work has been done in this regard, see the monographs and articles of Kilbas et al. [1], Miller and Ross [2], Podlubny [3], Samko

\section{Springer}

(c) 2012 Zhang and Gong; licensee Springer. This is an Open Access article distributed under the terms of the Creative Commons Attribution License (http://creativecommons.org/licenses/by/2.0), which permits unrestricted use, distribution, and reproduction in any medium, provided the original work is properly cited. 
et al. [4], Agarwal [5], Lakshmikantham [6] and Vasundhara Devi [7] and the references therein.

Recently, fractional differential equations have been of great interest, and boundary value problems for fractional differential equations have been considered by the use of techniques of nonlinear analysis (fixed-point theorems [8-10], Leray-Schauder theory $[11,12]$, lower and upper solution method, monotone iterative method [13-15]).

Variational methods have turned out to be a very effective analytical tool in the study of nonlinear problems. The classical critical point theory for $C^{1}$ functional was developed in the sixties and seventies (see $[16,17])$. The celebrated and important result in the last 30 years was the mountain pass theorem due to Ambrosetti and Rabinowitz [18] in 1973. The needs of specific applications (such as nonsmooth mechanics, nonsmooth gradient systems, etc.) and the impressive progress in nonsmooth analysis and multivalued analysis led to extensions of the critical point theory to nondifferentiable functions, locally Lipschitz functions in particular. The nonsmooth critical point theory for locally Lipschitz functions started with the work of Chang (see [19]). The theory of Chang was based on the subdifferential of locally Lipschitz functionals due to Clarke (see [20]). Using this subdifferential, Chang proposed a generalization of the well-known Palais-Smale condition and obtained various minimax principles concerning the existence and characterization of critical points for locally Lipschitz functions. Chang used his theory to study semilinear elliptic boundary value problems with a discontinuous nonlinearity. Later, in 2000, Kourogenis and Papageorgiou (see [21]) extended the theory of Chang and obtained some nonsmooth critical point theories and applied these to nonlinear elliptic equations at resonance, involving the $p$-Laplacian with discontinuous nonlinearities. Subsequently, many authors also studied the nonsmooth critical point theory (see [22-26]), then the nonsmooth critical point theory is also widely used to deal with nonlinear boundary value problems (see [27-31]). A good survey for nonsmooth critical point theory and nonlinear boundary value problems is the book of Gasinski and Papageorgiou [32].

There are some papers which are devoted to the boundary value problems for fractional differential inclusions (see [33-35]), and the main tools they use are fixed point theory for multi-valued contractions. However, to the best of the authors' knowledge, there are few results on the solutions to fractional BVP which were established by the nonsmooth critical point theory, since it is often very difficult to establish a suitable space and variational functional for fractional differential equations with boundary conditions. Recently, Jiao and Zhou [36] introduced some appropriate function spaces as their working space and set up a variational functional for the following system:

$$
\left\{\begin{array}{l}
-\frac{d}{d t}\left(\frac{1}{2} 0 D_{t}^{-\beta}\left(u^{\prime}(t)\right)+\frac{1}{2} t_{T}^{-\beta}\left(u^{\prime}(t)\right)\right)=\nabla F(t, u(t)), \quad \text { a.e. } t \in[0, T], \\
u(0)=u(T)=0
\end{array}\right.
$$

where $T>0,{ }_{0} D_{t}^{-\beta}$ and ${ }_{t} D_{T}^{-\beta}$ are the left and right Riemann-Liouville fractional integrals of order $0 \leq \beta<1$ respectively, and $F$ is continuously differentiable.

They give two existence results of solutions for the above system by using the least action principle and mountain pass theorem in critical point theory. It is easy to see that system (1.1) is a generalization to system (1.2), and it is interesting to ask whether the results in [36] hold true when the potential $F$ is just locally Lipschitz. But the main difficulty is the variational structure given in [36] cannot be applied to system (1.1) directly. So we have to 
find a new approach to solve this problem, and the main idea of the new approach comes from the inspiration of Theorem 2.7.3 and Theorem 2.7.5 in [20].

The structure of the paper is as follows. In the next section, for the convenience of readers, we present the mathematical background needed and the corresponding variational structure for system (1.1). In Section 3, using variational methods, we prove two existence theorems for the solutions of problem (1.1) which generalize the results in [36]. Finally, in Section 4, two examples are presented to illustrate our results.

\section{Preliminaries}

Definition 2.1 (Left and right Riemann-Liouville fractional integrals) Let $f$ be a function defined on $[a, b]$. The left and right Riemann-Liouville fractional integrals of order $\gamma$ for function $f$, denoted by ${ }_{a} D_{t}^{-\gamma} f(t)$ and ${ }_{t} D_{b}^{-\gamma} f(t)$ respectively, are defined by

$$
{ }_{a} D_{t}^{-\gamma} f(t)=\frac{1}{\Gamma(\gamma)} \int_{a}^{t}(t-s)^{\gamma-1} f(s) d s, \quad t \in[a, b], \gamma>0
$$

and

$$
{ }_{t} D_{b}^{-\gamma} f(t)=\frac{1}{\Gamma(\gamma)} \int_{t}^{b}(s-t)^{\gamma-1} f(s) d s, \quad t \in[a, b], \gamma>0
$$

provided the right-hand sides are pointwise defined on $[a, b]$, where $\Gamma$ is the gamma function.

Definition 2.2 (Left and right Riemann-Liouville fractional derivatives) Let $f$ be a function defined on $[a, b]$. The left and right Riemann-Liouville fractional derivatives of order $\gamma$ for function $f$, denoted by ${ }_{a} D_{t}^{\gamma} f(t)$ and ${ }_{t} D_{b}^{\gamma} f(t)$ respectively, are defined by

$$
{ }_{a} D_{t}^{-\gamma} f(t)=\frac{d^{n}}{d t^{n}}{ }_{a} D_{t}^{\gamma-n} f(t)=\frac{1}{\Gamma(n-\gamma)} \frac{d^{n}}{d t^{n}}\left(\int_{a}^{t}(t-s)^{n-\gamma-1} f(s) d s\right)
$$

and

$$
{ }_{t} D_{b}^{-\gamma} f(t)=(-1)^{n} \frac{d^{n}}{d t^{n}} D_{b}^{\gamma-n} f(t)=\frac{1}{\Gamma(n-\gamma)}(-1)^{n} \frac{d^{n}}{d t^{n}}\left(\int_{t}^{b}(s-t)^{n-\gamma-1} f(s) d s\right),
$$

where $t \in[a, b], n-1 \leq \gamma<n$ and $n \in \mathbb{N}$.

Definition 2.3 (Left and right Caputo fractional derivatives) Let $\gamma \geq 0$ and $n \in \mathbb{N}$.

(i) If $\gamma \in(n-1, n)$ and $f \in A C^{n}\left([a, b], \mathbb{R}^{N}\right)$, then the left and right Caputo fractional derivatives of order $\gamma$ for function $f$, denoted by ${ }_{a}^{c} D_{t}^{\gamma} f(t)$ and ${ }_{t}^{c} D_{b}^{\gamma} f(t)$ respectively, exist almost everywhere on $[a, b] .{ }_{a}^{c} D_{t}^{\gamma} f(t)$ and ${ }_{t}^{c} D_{b}^{\gamma} f(t)$ are represented by

$$
{ }_{a}^{c} D_{t}^{\gamma} f(t)={ }_{a} D_{t}^{\gamma-n} f^{(n)}(t)=\frac{1}{\Gamma(n-\gamma)}\left(\int_{a}^{t}(t-s)^{n-\gamma-1} f^{(n)}(s) d s\right)
$$

and

$$
{ }_{t}^{c} D_{b}^{\gamma} f(t)=(-1)^{n}{ }_{t} D_{b}^{\gamma-n} f^{(n)}(t)=\frac{(-1)^{n}}{\Gamma(n-\gamma)}\left(\int_{t}^{b}(s-t)^{n-\gamma-1} f^{(n)}(s) d s\right)
$$


respectively, where $t \in[a, b]$. In particular, if $0<\gamma<1$, then

$$
{ }_{a}^{c} D_{t}^{\gamma} f(t)={ }_{a} D_{t}^{\gamma-1} f^{\prime}(t)=\frac{1}{\Gamma(1-\gamma)}\left(\int_{a}^{t}(t-s)^{-\gamma} f^{\prime}(s) d s\right), \quad t \in[a, b]
$$

and

$$
{ }_{t}^{c} D_{b}^{\gamma} f(t)=(-1){ }_{t} D_{b}^{\gamma-1} f^{\prime}(t)=-\frac{1}{\Gamma(1-\gamma)}\left(\int_{t}^{b}(s-t)^{-\gamma} f^{\prime}(s) d s\right), \quad t \in[a, b] .
$$

(ii) If $\gamma=n-1$ and $f \in A C^{n-1}\left([a, b], \mathbb{R}^{N}\right)$, then ${ }_{a}^{c} D_{t}^{n-1} f(t)$ and ${ }_{t}^{c} D_{b}^{n-1} f(t)$ are represented by

$$
{ }_{a}^{c} D_{t}^{(n-1)} f(t)=f^{(n-1)}(t) \quad \text { and } \quad{ }_{t}^{c} D_{b}^{n-1} f(t)=(-1)^{(n-1)} f^{(n-1)}(t), \quad t \in[a, b] .
$$

In particular, ${ }_{a}^{c} D_{t}^{0} f(t)={ }_{t}^{c} D_{b}^{0} f(t)=f(t), t \in[a, b]$.

Property 2.1 ([36]) The left and right Riemann-Liouville fractional integral operators have the property of a semigroup, i.e.,

$$
{ }_{a} D_{t}^{-\gamma_{1}}\left({ }_{a} D_{t}^{-\gamma_{2}} f(t)\right)={ }_{a} D_{t}^{-\gamma_{1}-\gamma_{2}} f(t) \quad \text { and }{ }_{t} D_{b}^{-\gamma_{1}}\left({ }_{t} D_{b}^{-\gamma_{2}} f(t)\right)={ }_{t} D_{b}^{-\gamma_{1}-\gamma_{2}} f(t), \quad \forall \gamma_{1}, \gamma_{2}>0
$$

at any point $t \in[a, b]$ for a continuous function $f$ and for almost every point in $[a, b]$ if the function $f \in L^{1}\left([a, b], \mathbb{R}^{N}\right)$.

Definition 2.4 ([36]) Define $0<\alpha \leq 1$ and $1<p<\infty$. The fractional derivative space $E_{0}^{\alpha, p}$ is defined by the closure of $C_{0}^{\infty}\left([0, T], \mathbb{R}^{N}\right)$ with respect to the norm

$$
\|u\|_{\alpha, p}=\left(\int_{0}^{T}|u(t)|^{p} d t+\int_{0}^{T}\left|{ }_{0}^{c} D_{t}^{\alpha} u(t)\right|^{p} d t\right)^{1 / p} \quad \forall u \in E_{0}^{\alpha, p}
$$

where $C_{0}^{\infty}\left([0, T], \mathbb{R}^{N}\right)$ denotes the set of all functions $u \in C^{\infty}\left([0, T], \mathbb{R}^{N}\right)$ with $u(0)=$ $u(T)=0$. It is obvious that the fractional derivative space $E_{0}^{\alpha, p}$ is the space of functions $u \in L^{p}\left(0, T ; \mathbb{R}^{N}\right)$ having an $\alpha$-order Caputo fractional derivative ${ }_{0}^{c} D_{t}^{\alpha} u \in L^{p}\left(0, T ; \mathbb{R}^{N}\right)$ and $u(0)=u(T)=0$.

Proposition 2.1 ([36]) Let $0<\alpha \leq 1$ and $1<p<\infty$. The fractional derivative space $E_{0}^{\alpha, p}$ is a reflexive and separable Banach space.

Proposition 2.2 ([36]) Let $0<\alpha \leq 1$ and $1<p<\infty$. For all $u \in E_{0}^{\alpha, p}$, we have

$$
\|u\|_{L^{p}} \leq \frac{T^{\alpha}}{\Gamma(\alpha+1)}\left\|{ }_{0}^{c} D_{t}^{\alpha} u\right\|_{L^{p}}
$$

Moreover, if $\alpha>1 / p$ and $1 / p+1 / q=1$, then

$$
\|u\|_{\infty} \leq \frac{T^{\alpha-1 / p}}{\Gamma(\alpha)((\alpha-1) q+1)^{1 / q}}\left\|{ }_{0}^{c} D_{t}^{\alpha} u\right\|_{L^{p}} .
$$


According to (2.1), we can consider $E_{0}^{\alpha, p}$ with respect to the norm

$$
\|u\|_{\alpha, p}=\left\|{ }_{0}^{c} D_{t}^{\alpha} u\right\|_{L^{p}}=\left(\int_{0}^{T}\left|{ }_{0}^{c} D_{t}^{\alpha} u(t)\right|^{p} d t\right)^{1 / p} .
$$

Proposition 2.3 ([36]) Define $0<\alpha \leq 1$ and $1<p<\infty$. Assume that $\alpha>1 / p$ and the sequence $\left\{u_{k}\right\}$ converges weakly to $u$ in $E_{0}^{\alpha, p}$, i.e., $u_{k} \rightarrow u$. Then $u_{k} \rightarrow u$ in $C\left([0, T], \mathbb{R}^{N}\right)$, i.e., $\left\|u-u_{k}\right\|_{\infty} \rightarrow 0$, as $k \rightarrow \infty$.

In this paper, we treat BVP (1.1) in the Hilbert space $E^{\alpha}:=E_{0}^{\alpha, 2}$ with the equivalent norm defined in (2.3).

Proposition 2.4 ([36]) If $1 / 2<\alpha \leq 1$, then for any $u \in E^{\alpha}$, we have

$$
|\cos (\pi \alpha)|\|u\|_{\alpha}^{2} \leq-\int_{0}^{T}\left({ }_{0}^{c} D_{t}^{\alpha} u(t),{ }_{t}^{c} D_{T}^{\alpha} u(t)\right) d t \leq \frac{1}{|\cos (\pi \alpha)|}\|u\|_{\alpha}^{2} .
$$

In order to establish the variational structure for system (1.1), it is necessary to construct some appropriate function spaces. The Cartesian product space $L_{2}^{p}$ defined by

$$
L_{2}^{p}\left([0, T], \mathbb{R}^{N}\right)=L^{p}\left([0, T], \mathbb{R}^{N}\right) \times L^{p}\left([0, T], \mathbb{R}^{N}\right)
$$

is also a reflexive and separable Banach space with respect to the norm

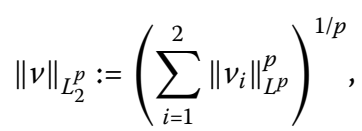

where $v=\left(v_{1}, v_{2}\right) \in L_{2}^{p}\left([0, T], \mathbb{R}^{N}\right)$.

The space $E_{p \times p}:=\left\{\left(u,{ }_{0}^{c} D_{t}^{\alpha} u\right): \forall u \in E_{0}^{\alpha, p}\right\}$ is a closed subset of $L_{2}^{p}\left([0, T], \mathbb{R}^{N}\right)$ under the norm (2.4) as $E_{0}^{\alpha, p}$ is closed by Definition 2.4.

In this paper, we use the norm defined in (2.3), which is an equivalent norm in $E_{p \times p}$ with norm (2.4).

Definition 2.5 Let $L^{\infty}\left([0, T], \mathbb{R}^{N} \times \mathbb{R}^{N}\right)$ denote the space of essentially bounded measurable functions from $[0, T]$ into $\mathbb{R}^{N} \times \mathbb{R}^{N}$ under the norm

$$
\|(u, v)\|_{L^{\infty}}:=\operatorname{ess} \sup \{|u(t)|+|v(t)|: t \in[0, T]\} .
$$

It is obvious that $L^{\infty}\left([0, T], \mathbb{R}^{N} \times \mathbb{R}^{N}\right)$ is a Banach space under the norm (2.5).

Remark 2.5 We use $E_{\infty}$ and $\|(u, v)\|_{E_{\infty}}$ to denote $L^{\infty}\left([0, T], \mathbb{R}^{N} \times \mathbb{R}^{N}\right)$ and $\|(u, v)\|_{L^{\infty}}$ respectively.

Definition 2.6 ([13]) Let $f$ be Lipschitz near a given point $x$ in a Banach space $X$, and $v$ be any other vector in $X$. The generalized directional derivative of $f$ at $x$ in the direction $v$, denoted by $f^{0}(x ; v)$, is defined as follows:

$$
f^{0}(x ; v)=\limsup _{y \rightarrow x, \lambda \downarrow 0} \frac{f(y+\lambda v)-f(y)}{\lambda},
$$


where $y$ is also a vector in $X$ and $\lambda$ is a positive scalar, and we denote by

$$
\partial f(x):=\left\{x^{*} \in X^{*}: f^{0}(x ; v) \geq\left\langle x^{*}, v\right\rangle, \text { for all } v \text { in } X\right\}
$$

the generalized gradient of $f$ at $x$ (the Clarke subdifferential).

Lemma 2.1 ([20]) Let $x$ and $y$ be points in a Banach space $X$, and suppose that is Lipschitz on an open set containing the line segment $[x, y]$. Then there exists a point $u$ in $(x, y)$ such that

$$
f(y)-f(x) \in\langle\partial f(u), y-x\rangle
$$

Definition 2.7 ([32]) A point $u \in X$ is said to be a critical point of a locally Lipschitz $f$ if $\theta \in \partial f(u)$, namely $f^{0}(u ; v) \geq 0$ for every $v \in X$. A real number $c$ is called a critical value of $f$ if there is a critical point $u \in X$ such that $f(u)=c$.

Definition 2.8 ([32]) If $f$ is a locally Lipschitz function, we say that $f$ satisfies the nonsmooth (P.S.) condition if each sequence $\left(x_{n}\right)$ in $X$ such that $f\left(x_{n}\right)$ is bounded and $\lim _{n \rightarrow \infty} \lambda\left(x_{n}\right)=0$ has a convergent subsequence, where $\lambda(x):=\min _{x^{*} \in \partial f(x)}\left\|x^{*}\right\|$.

Clarke considered the following abstract framework in [20]:

- let $(S, \mathcal{T}, \mu)$ be a $\sigma$-finite positive measure space, and let $Y$ be a separable Banach space;

- let $Z$ be a closed subspace of $L^{\infty}(S, Y)$, where $L^{\infty}(S, Y)$ denotes the space of measure essentially bounded functions mapping $S$ to $Y$, equipped with the usual supremum norm;

- define a functional $f$ on $Z$ via

$$
f(x)=\int_{S} f_{t}(x(t)) \mu(d t)
$$

where $Z$ is a closed subspace of $L^{\infty}(S, Y)$ and $f_{t}: Y \rightarrow \mathbb{R}(t \in S)$ is a given family of functions;

- suppose that the mapping $t \rightarrow f_{t}(v)$ is measurable for each $v$ in $Y$, and that $x$ is a point at which $f(x)$ is defined (finitely);

- suppose that there exist $\varepsilon>0$ and a function $k(t)$ in $L^{1}(S, \mathbb{R})$ such that

$$
\left|f_{t}\left(v_{1}\right)-f_{t}\left(v_{2}\right)\right| \leq k(t)\left\|v_{1}-v_{2}\right\|_{Y}
$$

for all $t \in S$ and all $v_{1}$ and $v_{2}$ in $x(t)+\varepsilon B_{Y}$.

Under the conditions described above, $f$ is Lipschitz in a neighborhood of $x$ and one has

$$
\partial f(x) \subset \int_{S} \partial f_{t}(x(t)) \mu(d t) .
$$

Further, if each $f_{t}$ is regular at $x(t)$ for each $t$, then $f$ is regular at $x$ and the equality holds. 
Remark 2.6 The interpretation of (2.7) is as follows: To every $\zeta \in \partial f(x)$, there is a corresponding mapping $t \rightarrow \zeta_{t}$ from $S$ to $Y^{*}$ with

$$
\zeta_{t} \in \partial f_{t}(x(t)) \quad \text { for a.e. } t \in \text { relative to the measure } \mu \text {, }
$$

and having the property that for every $v$ in $Z$, one has

$$
\langle\zeta, v\rangle=\int_{S}\left\langle\zeta_{t}, v(t)\right\rangle \mu(d t)
$$

Thus, every $\zeta$ in the left-hand side of (2.7) is an element of $Z$ that can be written

$$
\zeta(\cdot)=\int_{S}\left\langle\zeta_{t}, \cdot\right\rangle \mu(d t)
$$

where $t \rightarrow \zeta_{t}$ is a measurable selection of $\partial f_{t}(x(t))$.

Lemma 2.2 Let $F$ satisfy the condition (A) and $L:[0, T] \times \mathbb{R}^{N} \times \mathbb{R}^{N} \rightarrow \mathbb{R}$ be given by $L(t, x, y)=-F(t, x)$, then define a functional $f$ on $E_{2 \times 2}$ by

$$
f\left(u,{ }_{0}^{c} D_{t}^{\alpha} u\right)=\int_{0}^{T} L\left(t, u(t),{ }_{0}^{c} D_{t}^{\alpha} u(t)\right) d t=\int_{0}^{T}-F(t, u(t)) d t .
$$

Then $f$ is Lipschitz on $E_{2 \times 2}$, and one has

$$
\partial f\left(u,{ }_{0}^{c} D_{t}^{\alpha} u\right) \subset \int_{0}^{T}\{\partial(-F(t, u(t)))\} \times\{0\} d t .
$$

Proof Take an arbitrary element $\left(u_{0},{ }_{0}^{c} D_{t}^{\alpha} u_{0}\right)$ in $E_{2 \times 2}$, then it suffices to prove $f$ is Lipschitz on $\left(u_{0},{ }_{0}^{c} D_{t}^{\alpha} u_{0}\right)$.

When $\left\|\left(u_{i},{ }_{0}^{c} D_{t}^{\alpha} u_{i}\right)-\left(u_{0},{ }_{0}^{c} D_{t}^{\alpha} u_{0}\right)\right\|_{E_{2 \times 2}} \leq \varepsilon(i=1,2)$, we conclude

$$
\left\|u_{i}-u_{0}\right\|_{\infty} \leq C_{1} \varepsilon
$$

by Proposition 2.2, where $C_{1}:=\frac{T^{\alpha-1 / 2}}{\Gamma(\alpha)(2 \alpha-1)^{1 / 2}}$. In view of Lemma 2.1 and (A), one has

$$
\left|-F\left(t, u_{1}(t)\right)+F\left(t, u_{2}(t)\right)\right| \leq k^{\prime}(t)\left|u_{1}(t)-u_{2}(t)\right|
$$

for a.e. $t \in[0, T]$, where $k^{\prime}(t) \in L^{1}\left(0, T ; \mathbb{R}^{+}\right)$.

By (2.9) and (2.10), we have

$$
\begin{aligned}
\left|f\left(u_{1},{ }_{0}^{c} D_{t}^{\alpha} u_{1}\right)-f\left(u_{2},{ }_{0}^{c} D_{t}^{\alpha} u_{2}\right)\right| & =\left|\int_{0}^{T}-F\left(t, u_{1}(t)\right)+F\left(t, u_{2}(t)\right) d t\right| \\
& \leq\left(\int_{0}^{T} k^{\prime}(t) d t\right)\left\|u_{1}-u_{2}\right\|_{\infty} \\
& \leq C_{1} \int_{0}^{T} k^{\prime}(t) d t\left\|\left(u_{1},{ }_{0}^{c} D_{t}^{\alpha} u_{1}\right)-\left(u_{2},{ }_{0}^{c} D_{t}^{\alpha} u_{2}\right)\right\|_{E_{2 \times 2}},
\end{aligned}
$$

so $f$ is also Lipschitz on $\left(u_{0},{ }_{0}^{c} D_{t}^{\alpha} u_{0}\right)$. 
For any $\zeta$ in $\partial f\left(u_{0},{ }_{0}^{c} D_{t}^{\alpha} u_{0}\right)$, one has

$$
\int_{0}^{T}(-F)^{0}\left(t, u_{0}(t) ; v(t)\right) d t \geq f^{0}\left(\left(u_{0},{ }_{0}^{c} D_{t}^{\alpha} u_{0}\right) ;\left(v,{ }_{0}^{c} D_{t}^{\alpha} v\right)\right) \geq\left\langle\zeta,\left(v,{ }_{0}^{c} D_{t}^{\alpha} v\right)\right\rangle
$$

for any $\left(v,{ }_{0}^{c} D_{t}^{\alpha} v\right)$ in $E_{2 \times 2}$ by Fatou's lemma, and it is obvious that

$$
L^{0}\left(t, u_{0}(t),{ }_{0}^{c} D_{t}^{\alpha} u_{0}(t) ; v_{1}, v_{2}\right)=(-F)^{0}\left(t, u_{0}(t) ; v_{1}\right)
$$

for a.e. $t \in[0, T]$ and all $\left(v_{1}, v_{2}\right)$ in $\mathbb{R}^{N} \times \mathbb{R}^{N}$. Then we conclude

$$
\begin{aligned}
& \int_{0}^{T} L^{0}\left(t, u_{0}(t),{ }_{0}^{c} D_{t}^{\alpha} u_{0}(t) ; v(t),{ }_{0}^{c} D_{t}^{\alpha} v(t)\right) d t \\
& \quad \geq f^{0}\left(\left(u_{0},{ }_{0}^{c} D_{t}^{\alpha} u_{0}\right) ;\left(v,{ }_{0}^{c} D_{t}^{\alpha} v\right)\right) \geq\left\langle\zeta,\left(v,{ }_{0}^{c} D_{t}^{\alpha} v\right)\right\rangle,
\end{aligned}
$$

by (2.12) for any $\left(v,{ }_{0}^{c} D_{t}^{\alpha} v\right)$ in $E_{2 \times 2}$ and (2.13) remains true if we restrict $\left(\nu,{ }_{0}^{c} D_{t}^{\alpha} v\right)$ to $E_{2 \times 2} \cap$ $E_{\infty}$, which is a closed subspace of $E_{\infty}$ by Definition 2.4. The bounded linear functional $\zeta$ on $E_{2 \times 2}$ restricted to $E_{2 \times 2} \cap E_{\infty}$ is also a bounded linear functional, and we use $\zeta^{\prime}$ to denote the functional restricted on $E_{2 \times 2} \cap E_{\infty}$.

We interpret $(2.13)$ by saying that $\zeta^{\prime}$ belongs to the subgradient at $(0,0)$ of the convex functional

$$
\hat{f}\left(v,{ }_{0}^{c} D_{t}^{\alpha} v\right):=\int_{0}^{T} \hat{f}_{t}\left(v(t),{ }_{0}^{c} D_{t}^{\alpha} v(t)\right) d t,
$$

which is defined in $E_{2 \times 2} \cap E_{\infty}$, where $\hat{f}_{t}\left(v_{1}, v_{2}\right):=L_{2}^{0}\left(t, u_{0}(t),{ }_{0}^{c} D_{t}^{\alpha} u_{0}(t) ; v_{1}, v_{2}\right)$ for all $\left(v_{1}, v_{2}\right)$ in $\mathbb{R}^{N} \times \mathbb{R}^{N}$. In view of condition (A) and (2.12), we have

$$
\begin{aligned}
& \left|L_{2}^{0}\left(t, u_{0}(t),{ }_{0}^{c} D_{t}^{\alpha} u_{0}(t) ; v_{1}, v_{2}\right)-L_{2}^{0}\left(t, u_{0}(t),{ }_{0}^{c} D_{t}^{\alpha} u_{0}(t) ; v_{3}, v_{4}\right)\right| \\
& \quad=\left|(-F)^{0}\left(t, u_{0}(t) ; v_{1}\right)-(-F)^{0}\left(t, u_{0}(t) ; v_{3}\right)\right| \\
& \quad \leq\left(f(t)\left|u_{0}(t)\right|^{v}+g(t)\right)\left|v_{1}-v_{3}\right| \\
& \quad \leq\left(f(t)\left|u_{0}(t)\right|^{v}+g(t)\right)\left(\left|v_{1}-v_{3}\right|+\left|v_{2}-v_{4}\right|\right)
\end{aligned}
$$

for a.e. $t \in[0, T]$ and all $\left(v_{1}, v_{2}\right),\left(v_{3}, v_{4}\right)$ in $\mathbb{R}^{N} \times \mathbb{R}^{N}$.

Now we can apply Clarke's abstract framework to $\hat{f}$ with the following cast of characters:

- $(T, \mathcal{T}, \mu):=[0, T]$ with the Lebesgue measure, and let $Y:=\mathbb{R}^{N} \times \mathbb{R}^{N}$, which is a separable Banach space with the norm $|\cdot|+|\cdot|$;

- let $Z:=E_{2 \times 2} \cap E_{\infty}$, which is a closed subspace of $E_{\infty}$, and $E_{\infty}$ denotes the space of measure essentially bounded functions mapping $T$ to $Y$, equipped with the usual supremum norm by Definition 2.5;

- define a functional $\hat{f}$ on $Z$ by (2.14);

- the mapping $t \rightarrow L^{0}\left(t, u_{0}(t),{ }_{0}^{c} D_{t}^{\alpha} u_{0}(t) ; v_{1}, v_{2}\right)$ is measurable for each $\left(v_{1}, v_{2}\right)$ in $\mathbb{R}^{N} \times \mathbb{R}^{N}$ (see [20]), and that $(0,0)$ is a point at which $\hat{f}$ is defined (finitely);

- the condition (2.6) in Clarke's abstract framework is satisfied by (2.15).

By (2.12), we get

$$
\partial \hat{f}_{t}(0,0)=\partial L\left(t, u_{0}(t),{ }_{0}^{c} D_{t}^{\alpha} u_{0}(t)\right) \subset \partial\left(-F\left(t, u_{0}(t)\right)\right) \times\{0\},
$$


thus, every $\zeta^{\prime} \in \partial \hat{f}(0,0)$ can be written as

$$
\begin{aligned}
\left\langle\zeta^{\prime},\left(v,{ }_{0}^{c} D_{t}^{\alpha} v\right)\right\rangle & =\int_{0}^{T}(q(t), v(t))+\left(0,{ }_{0}^{c} D_{t}^{\alpha} v(t)\right) d t \\
& =\int_{0}^{T}(q(t), v(t)) d t
\end{aligned}
$$

for any $\left(v,{ }_{0}^{c} D_{t}^{\alpha} v\right)$ to $E_{2 \times 2} \cap E_{\infty}$, where $q(t) \in \partial\left(-F\left(t, u_{0}(t)\right)\right)$ for a.e. $t \in[0, T]$.

When $v \in C_{0}^{\infty}\left([0, T], \mathbb{R}^{N}\right)$, it is obvious that $\left(v,{ }_{0}^{c} D_{t}^{\alpha} v\right) \in E_{2 \times 2} \cap E_{\infty}$ and $\left(v,{ }_{0}^{c} D_{t}^{\alpha} v\right)$ is dense in $E_{2 \times 2}$ by Definition 2.4. So for each $\left(v,{ }_{0}^{c} D_{t}^{\alpha} v\right) \in E_{2 \times 2}$, we can choose $\left(v_{n},{ }_{0}^{c} D_{t}^{\alpha} v_{n}\right) \in E_{2 \times 2} \cap$ $E_{\infty}$ such that

$$
\left\|\left(v_{n},{ }_{0}^{c} D_{t}^{\alpha} v_{n}\right)-\left(v,{ }_{0}^{c} D_{t}^{\alpha} v\right)\right\|_{E_{2 \times 2}} \rightarrow 0 \quad \text { and } \quad\left\langle\zeta,\left(v_{n},{ }_{0}^{c} D_{t}^{\alpha} v_{n}\right)\right\rangle \rightarrow\left\langle\zeta,\left(v,{ }_{0}^{c} D_{t}^{\alpha} v\right)\right\rangle \text {. }
$$

Combining (2.16) and (2.17), we have

$$
\left\langle\zeta,\left(v,{ }_{0}^{c} D_{t}^{\alpha} v\right)\right\rangle=\int_{0}^{T}(q(t), v(t)) d t
$$

for all $\left(v,{ }_{0}^{c} D_{t}^{\alpha} v\right) \in E_{2 \times 2}$. Then we conclude

$$
\partial f\left(u_{0},{ }_{0}^{c} D_{t}^{\alpha} u_{0}\right) \subset \int_{0}^{T}\left\{\partial\left(-F\left(t, u_{0}(t)\right)\right)\right\} \times\{0\} d t
$$

and this completes the proof.

Remark 2.7 The interpretation of expression (2.8) is as follows: If $\left(u_{0},{ }_{0}^{c} D_{t}^{\alpha} u_{0}\right)$ is an element in $E_{2 \times 2}$ and $\zeta \in \partial f\left(u_{0},{ }_{0}^{c} D_{t}^{\alpha} u_{0}\right)$, we deduce the existence of a measurable function $(r(t), s(t))$ such that

$$
r(t) \in \partial\left(-F\left(t, u_{0}(t)\right)\right) \quad \text { and } \quad s(t)=0
$$

for a.e. $t \in[0, T]$ and one has

$$
\left\langle\zeta,\left(v,{ }_{0}^{c} D_{t}^{\alpha} v\right)\right\rangle=\int_{0}^{T}\langle r(t), v(t)\rangle+\left\langle 0,{ }_{0}^{c} D_{t}^{\alpha} v(t)\right\rangle d t
$$

and for any $\left(v,{ }_{0}^{c} D_{t}^{\alpha} v\right)$ in $E_{2 \times 2}$.

Define a functional $\phi$ on $E^{\alpha}$ by

$$
\phi(u)=-\int_{0}^{T} F(t, u(t)) d t,
$$

if $\eta \in \partial f\left(u_{0},{ }_{0}^{c} D_{t}^{\alpha} u_{0}\right)$ on $E_{2 \times 2}$, then we can define $\eta^{\prime}$ on $E^{\alpha}$ by

$$
\eta^{\prime}(u):=\eta\left(u,{ }_{0}^{c} D_{t}^{\alpha} u\right),
$$

for all $u \in E^{\alpha}$, it is easy to verify $\eta^{\prime} \in \partial \phi\left(u_{0}\right)$. 
Similarly, if $\xi \in \partial \phi\left(u_{0}\right)$ on $E^{\alpha}$, then we can define $\xi^{\prime}$ on $E_{2 \times 2}$ by

$$
\xi^{\prime}\left(u,{ }_{0}^{c} D_{t}^{\alpha} u\right):=\xi(u)
$$

for all $\left(u,{ }_{0}^{c} D_{t}^{\alpha} u\right) \in E_{2 \times 2}$, and it is easy to verify $\xi^{\prime} \in \partial f\left(u_{0},{ }_{0}^{c} D_{t}^{\alpha} u_{0}\right)$.

Lemma 2.3 The corresponding functionals $\varphi_{1}$ and $\varphi_{2}$ on $E^{\alpha}$ are given by

$$
\varphi_{1}(u)=-\frac{1}{2} \int_{0}^{T}\left({ }_{0}^{c} D_{t}^{\alpha} u(t),{ }_{t}^{c} D_{T}^{\alpha} u(t)\right) d t
$$

and

$$
\varphi_{2}(u)=\int_{0}^{T}-F(t, u(t)) d t
$$

where $F$ satisfies the condition (A) and $1 / 2<\alpha \leq 1$, then the functional defined by

$$
\begin{aligned}
\varphi(u) & =\varphi_{1}(u)+\varphi_{2}(u) \\
& =\int_{0}^{T}\left[-\frac{1}{2}\left({ }_{0}^{c} D_{t}^{\alpha} u(t),{ }_{t}^{c} D_{T}^{\alpha} u(t)\right)-F(t, u(t))\right] d t,
\end{aligned}
$$

is Lipschitz on $E^{\alpha}$, and $\forall u, v \in E^{\alpha}$, we have

$$
\langle\varsigma, v\rangle=-\int_{0}^{T} \frac{1}{2}\left[\left({ }_{0}^{c} D_{t}^{\alpha} u(t),{ }_{t}^{c} D_{T}^{\alpha} v(t)\right)+\left({ }_{t}^{c} D_{T}^{\alpha} u(t),{ }_{0}^{c} D_{t}^{\alpha} v(t)\right)\right] d t+\int_{0}^{T}(q(t), v(t)) d t
$$

where $\varsigma \in \partial \varphi(u)$ and $q(t) \in \partial(-F(t, u(t)))$.

Proof By direct computation, it is obvious that

$$
\left\langle\varphi_{1}^{\prime}(u), v\right\rangle=-\int_{0}^{T} \frac{1}{2}\left[\left({ }_{0}^{c} D_{t}^{\alpha} u(t),{ }_{t}^{c} D_{T}^{\alpha} v(t)\right)+\left({ }_{t}^{c} D_{T}^{\alpha} u(t),{ }_{0}^{c} D_{t}^{\alpha} v(t)\right)\right] d t .
$$

In view of Lemma 2.2 and Remark 2.7, if $\tau \in \partial \varphi_{2}(u)$, then we have

$$
\langle\tau, v\rangle=\int_{0}^{T}(q(t), v(t)) d t
$$

where $q(t) \in \partial(-F(t, u(t)))$.

Since $\partial \varphi(u) \subset \partial \varphi_{1}(u)+\partial \varphi_{2}(u)$, (2.21) holds by (2.22) and (2.23), and this completes the proof.

Making use of Property 2.1 and Definition 2.3, for any $u \in A C\left([0, T], \mathbb{R}^{N}\right)$, BVP (1.1) is equivalent to the following problem:

$$
\left\{\begin{array}{l}
-\frac{d}{d t}\left(\frac{1}{2}{ }_{0} D_{t}^{\alpha-1}\left({ }_{0}^{c} D_{t}^{\alpha} u(t)\right)+\frac{1}{2} t D_{T}^{\alpha-1}\left({ }_{t}^{c} D_{T}^{\alpha} u(t)\right)\right) \in \partial F(t, u(t)), \quad \text { a.e. } t \in[0, T] \\
u(0)=u(T)=0
\end{array}\right.
$$


where $\alpha=1-\beta / 2 \in(1 / 2,1]$. Therefore, we seek a solution $u$ of BVP (2.24), which corresponds to the solution $u$ of BVP (1.1) provided that $u \in A C\left([a, b], \mathbb{R}^{N}\right)$.

Let us denote $D^{\alpha}(u(t))$ by

$$
D^{\alpha}(u(t))=\frac{1}{2}{ }_{0} D_{t}^{\alpha-1}\left({ }_{0}^{c} D_{t}^{\alpha} u(t)\right)+\frac{1}{2}{ }_{t} D_{T}^{\alpha-1}\left({ }_{t}^{c} D_{T}^{\alpha} u(t)\right)
$$

then we are in a position to give the definition of the solution of BVP (2.24).

Definition 2.9 A function $u \in A C\left([0, T], \mathbb{R}^{N}\right)$ is called a solution of BVP (2.24) if

(i) $D^{\alpha}(u(t))$ is differentiable for almost every $t \in[0, T]$.

(ii) $u$ satisfies (2.24).

Lemma 2.4 Let $1 / 2<\alpha \leq 1$, and $\varphi$ is defined by (2.20). If assumption (A) is satisfied and $u \in E^{\alpha}$ is a solution of the corresponding Euler equation $0 \in \partial \varphi(u)$, then $u$ is a solution of $B V P$ (2.24) which, of course, corresponds to the solution of BVP (1.1).

Proof By Lemma 2.3, we have

$$
\begin{aligned}
0= & -\int_{0}^{T} \frac{1}{2}\left[\left({ }_{0}^{c} D_{t}^{\alpha} u(t),{ }_{t}^{c} D_{T}^{\alpha} v(t)\right)+\left({ }_{t}^{c} D_{T}^{\alpha} u(t),{ }_{0}^{c} D_{t}^{\alpha} v(t)\right)\right] d t \\
& -\int_{0}^{T}(q(t), v(t)) d t \\
= & \int_{0}^{T} \frac{1}{2}\left({ }_{0} D_{t}^{\alpha-1}\left({ }_{0}^{c} D_{t}^{\alpha} u(t)\right), v^{\prime}(t)\right)-\frac{1}{2}\left({ }_{t} D_{T}^{\alpha-1}\left({ }_{t}^{c} D_{T}^{\alpha} u(t)\right), v^{\prime}(t)\right) d t \\
& -\int_{0}^{T}(q(t), v(t)) d t,
\end{aligned}
$$

where for all $v \in E^{\alpha}$ and $q(t) \in \partial F(t, u(t))$.

Let us define $\omega \in C\left([0, T], \mathbb{R}^{N}\right)$ by

$$
w(t)=\int_{0}^{t} q(s) d s, \quad t \in[0, T]
$$

so that

$$
\int_{0}^{T}\left(w(t), v^{\prime}(t)\right) d t=\int_{0}^{T}\left[\int_{0}^{t}\left(q(t), v^{\prime}(t)\right) d s\right] d t .
$$

By the Fubini theorem and noting that $v(T)=0$, we obtain

$$
\begin{aligned}
\int_{0}^{T}\left(w(t), v^{\prime}(t)\right) d t & =\int_{0}^{T}\left[\int_{s}^{T}\left(q(t), v^{\prime}(t)\right) d t\right] d s \\
& =\int_{0}^{T}(q(s), v(T)-v(s)) d s \\
& =-\int_{0}^{T}(q(s), v(T)-v(s)) d s .
\end{aligned}
$$


Hence, by (2.26) we have, for every $v \in E^{\alpha}$,

$$
\int_{0}^{T}\left(\frac{1}{2}{ }_{0} D_{t}^{\alpha-1}\left({ }_{0}^{c} D_{t}^{\alpha} u(t)\right)-\frac{1}{2}{ }_{t} D_{T}^{\alpha-1}\left({ }_{t}^{c} D_{T}^{\alpha} u(t)\right)+\omega(t), v^{\prime}(t)\right) d t=0 .
$$

If $\left(e_{j}\right)$ denotes the Canonical basis of $\mathbb{R}^{N}$, we can choose $v \in E^{\alpha}$ such that

$$
v(t)=\sin \frac{2 k \pi}{T} e_{j} \quad \text { or } \quad v(t)=e_{j}-\cos \frac{2 k \pi}{T} e_{j}, \quad k=1, \ldots \text { and } j=1, \ldots, N
$$

The theory of Fourier series and (2.27) imply that

$$
\frac{1}{2}{ }_{0} D_{t}^{\alpha-1}\left({ }_{0}^{c} D_{t}^{\alpha} u(t)\right)-\frac{1}{2}{ }_{t} D_{T}^{\alpha-1}\left({ }_{t}^{c} D_{T}^{\alpha} u(t)\right)+\omega(t)=C
$$

a.e. on $[0, T]$ for some $C \in \mathbb{R}^{N}$. According to the definition of $\omega \in C\left([0, T], \mathbb{R}^{N}\right)$, we have

$$
\frac{1}{2}{ }_{0} D_{t}^{\alpha-1}\left({ }_{0}^{c} D_{t}^{\alpha} u(t)\right)-\frac{1}{2}{ }_{t} D_{T}^{\alpha-1}\left({ }_{t}^{c} D_{T}^{\alpha} u(t)\right)=-\int_{0}^{t} q(s) d s+C
$$

a.e. on $[0, T]$ for some $C \in \mathbb{R}^{N}$.

In view of $q(t) \in L^{1}\left([0, T], \mathbb{R}^{N}\right)$, we shall identify the equivalence class $D^{\alpha}(u(t))$ given by its continuous representant

$$
D^{\alpha}(u(t))=\frac{1}{2}{ }_{0} D_{t}^{\alpha-1}\left({ }_{0}^{c} D_{t}^{\alpha} u(t)\right)-\frac{1}{2}{ }_{t} D_{T}^{\alpha-1}\left({ }_{t}^{c} D_{T}^{\alpha} u(t)\right)=-\int_{0}^{t} q(s) d s+C
$$

for $t \in[0, T]$.

Therefore, it follows from (2.28) and the classical result of the Lebesgue theory that $q(t)$ is the classical derivative of $D^{\alpha}(u(t))$ a.e. on $[0, T]$ which means that (i) in Definition 2.9 is verified.

Since $u \in E^{\alpha}$ implies that $u \in A C\left([0, T], \mathbb{R}^{N}\right)$, it remains to show that $u$ satisfies (2.24). In fact, according to (2.29), we can get that

$$
\frac{d}{d t} D^{\alpha}(u(t))=\frac{d}{d t}\left(\frac{1}{2}{ }_{0} D_{t}^{\alpha-1}\left({ }_{0}^{c} D_{t}^{\alpha} u(t)\right)-\frac{1}{2}{ }_{t} D_{T}^{\alpha-1}\left({ }_{t}^{c} D_{T}^{\alpha} u(t)\right)\right)=-q(t) .
$$

Moreover, $u \in E^{\alpha}$ implies that $u(0)=u(T)=0$.

Lemma 2.5 ([32]) Let $X$ be a real reflexive Banach space. If the functional $\psi: H \rightarrow \mathbb{R}$ is weakly lower semi-continuous and coercive, i.e., $\lim _{\|z\| \rightarrow \infty} \psi(z)=+\infty$, then there exists $z_{0} \in H$ such that $\psi\left(z_{0}\right)=\inf _{z \in H} \psi(z)$. Moreover, then $\theta \in \partial \psi\left(z_{0}\right)$.

Lemma 2.6 ([32]) Let $X$ be a real reflexive Banach space, and $\psi: X \rightarrow \mathbb{R}$ is a locally Lipschitz function. If there exist $x_{1} \in X$ and $r>0$ such that $\left\|x_{1}\right\|_{X}>r$,

$$
\max \left\{\psi(0), \psi\left(x_{1}\right)\right\} \leq \inf _{\|x\|_{X}=r} \psi(x)
$$

and $\psi$ satisfies the nonsmooth (P.S.) condition with

$$
c:=\inf _{\gamma \in \Gamma} \sup _{t \in[0,1]} \psi(\gamma(t)),
$$


where

$$
\Gamma:=\left\{\gamma \in C([0,1] ; X): \gamma(0)=0, \gamma(1)=x_{1}\right\}
$$

Then $c \geq \inf _{\|x\|_{X}=r} \psi(x)$ and $c$ is a critical value of $\psi$.

Definition 2.10 ([37]) Assume that the compact group $G$ acts diagonally on $V^{k}$, that is,

$$
g\left(v_{1}, \ldots, v_{k}\right):=\left(g v_{1}, \ldots, g v_{k}\right)
$$

where $V$ is a finite dimensional space. The action of $G$ is admissible if every continuous equivariant map $\partial U \rightarrow V^{k-1}$ has a zero, where $U$ is an open bounded invariant neighborhood of 0 in $V^{k}, k \geq 2$.

Example 2.1 The antipodal action of $G:=Z_{2}$ on $V:=\mathbb{R}$ is admissible.

We consider the following situation:

(A1) The compact group $G$ acts isometrically on the Banach space $\overline{\bigoplus_{j \in \mathbb{N}} X_{j}}$, the space $X_{j}$ is invariant and there exists a finite dimensional space $V$ such that for each $j \in \mathbb{N}, X_{j} \simeq V$ and the action of $G$ on $V$ is admissible.

Lemma 2.7 ([27]) Suppose $\varphi: X \rightarrow \mathbb{R}$ is an invariant locally Lipschitz functional. If, for every $k \in \mathbb{N}$, there exist $\rho_{k}>r_{k}>0$ such that

(A2) $a_{k}:=\max _{u \in Y_{k},\|u\|=\rho_{k}} \varphi(u) \leq 0$, where $Y_{k}:=\bigoplus_{j=0}^{k} X_{j}$;

(A3) $b_{k}:=\inf _{u \in Z_{k},\|u\|=r_{k}} \varphi(u) \rightarrow \infty$, as $k \rightarrow \infty$, where $Z_{k}:=\overline{\bigoplus_{j=k}^{\infty} X_{j}}$;

(A4) $\varphi$ satisfies the nonsmooth (P.S.) $)_{c}$ condition for every $c>0$.

Then $\varphi$ has an unbounded sequence of critical values.

Remark 2.8 The condition (A1) is needed for the proof of Lemma 2.7, see details in [27] and the references therein.

\section{Main results and proofs of the theorems}

Theorem 3.1 Let $\alpha \in(1 / 2,1]$ and F satisfy the condition (A), and suppose the following conditions hold:

(B1) there exist $\beta>2$ and $r_{1}>0$ such that

$$
F^{0}(t, x ;-x) \leq-\beta F(t, x)
$$

for a.e. $t \in[0, T]$ and all $|x| \geq r_{1}$ in $\mathbb{R}^{N}$;

(B2) $\int_{0}^{T} F(t, 0) d t=0$ and

$$
\limsup _{|x| \rightarrow 0} \frac{F(t, x)}{|x|^{2}} \leq \frac{\Gamma^{2}(\alpha+1)}{2 T^{2 \alpha}}
$$

uniformly for a.e. $t \in[0, T]$; 
(B3) there exist $\mu>2$ and $Q>0$ such that

$$
\liminf _{|x| \rightarrow \infty} \frac{F(t, x)}{|x|^{\mu}} \geq Q
$$

uniformly for a.e. $t \in[0, T]$.

Then system (1.1) has at least one solution on $E^{\alpha}$.

Proof Let $\left\{u_{n}\right\} \subset E^{\alpha}$ such that $\varphi\left(u_{n}\right)$ is bounded and $\lambda\left(u_{n}\right) \rightarrow 0$ as $n \rightarrow \infty$. First, we prove $\left\{u_{n}\right\}$ is a bounded sequence. Take $u_{n}^{*} \in \partial \varphi\left(u_{n}\right)$ such that $\lambda\left(u_{n}\right)=\left\|u_{n}^{*}\right\|$, then there exists $q_{n}(t) \in \partial\left(-F\left(t, u_{n}(t)\right)\right)$ such that

$$
\left\langle u_{n}^{*}, u_{n}\right\rangle=-\int_{0}^{T}\left({ }_{0}^{c} D_{t}^{\alpha} u_{n}(t),{ }_{t}^{c} D_{T}^{\alpha} u_{n}(t)\right) d t+\int_{0}^{T}\left(q_{n}(t), u_{n}(t)\right) d t
$$

for all $v \in E^{\alpha}$. It follows from (3.1) that

$$
\begin{aligned}
&\left(\frac{\beta}{2}-1\right)|\cos (\pi \alpha)|\left\|u_{n}\right\|_{\alpha}^{2} \\
& \leq \int_{0}^{T}\left(1-\frac{\beta}{2}\right)\left({ }_{0}^{c} D_{t}^{\alpha} u_{n}(t),{ }_{t}^{c} D_{T}^{\alpha} u_{n}(t)\right) d t \\
&=\beta \varphi\left(u_{n}\right)-\left\langle u_{n}^{*}, u_{n}\right\rangle+\int_{0}^{T}\left[\beta F\left(t, u_{n}(t)\right)+\left(q_{n}(t), u_{n}(t)\right)\right] d t \\
&=\beta \varphi\left(u_{n}\right)-\left\langle u_{n}^{*}, u_{n}\right\rangle+\int_{\Omega_{1}}\left[\beta F\left(t, u_{n}(t)\right)+\left(q_{n}(t), u_{n}(t)\right)\right] d t+\int_{\Omega_{2}}\left[\beta F\left(t, u_{n}(t)\right)\right. \\
&\left.+\left(q_{n}(t), u_{n}(t)\right)\right] d t \\
& \leq \beta \varphi\left(u_{n}\right)-\left\langle u_{n}^{*}, u_{n}\right\rangle+\int_{\Omega_{1}}\left[\beta F\left(t, u_{n}(t)\right)+\left(q_{n}(t), u_{n}(t)\right)\right] d t+\int_{\Omega_{2}}\left[\beta F\left(t, u_{n}(t)\right)\right. \\
&\left.+(-F)^{0}\left(t, u_{n}(t) ; u_{n}(t)\right)\right] d t \\
&= \beta \varphi\left(u_{n}\right)-\left\langle u_{n}^{*}, u_{n}\right\rangle+\int_{\Omega_{1}}\left[\beta F\left(t, u_{n}(t)\right)+\left(q_{n}(t), u_{n}(t)\right)\right] d t+\int_{\Omega_{2}}\left[\beta F\left(t, u_{n}(t)\right)\right. \\
&\left.+F^{0}\left(t, u_{n}(t) ;-u_{n}(t)\right)\right] d t \\
& \leq \beta \varphi\left(u_{n}\right)-\left\langle u_{n}^{*}, u_{n}\right\rangle+\int_{\Omega_{1}}\left[\beta F\left(t, u_{n}(t)\right)+\left(q_{n}(t), u_{n}(t)\right)\right] d t,
\end{aligned}
$$

where $\Omega_{1}:=\left\{t \in[0, T] ;\left|u_{n}(t)\right| \leq r_{1}\right\}$ and $\Omega_{2}:=[0, T] \backslash \Omega_{1}$.

By (A) and the nonsmooth (P.S.) condition, we have

$$
\beta \varphi\left(u_{n}\right)+\int_{\Omega_{1}}\left[\beta F\left(t, u_{n}(t)\right)+\left(q_{n}(t), u_{n}(t)\right)\right] d t
$$

is bounded, which combined with (3.2) implies that $\left\{u_{n}\right\}$ is bounded in $E^{\alpha}$ since $\beta>2$.

By Proposition 2.3, the sequence $\left\{u_{n}\right\}$ has a subsequence, also denoted by $\left\{u_{n}\right\}$, such that

$$
u_{n} \rightarrow u \quad \text { weakly in } E^{\alpha} \quad \text { and } \quad u_{n} \rightarrow u \quad \text { strongly in } C\left([0, T] ; \mathbb{R}^{N}\right)
$$

and $\left\|u_{n}\right\|_{\infty} \leq C_{2}$ is bounded, where $C_{2}$ is a positive constant. 
Therefore, we have $u_{n}^{*} \in \partial \varphi\left(u_{n}\right)$, where $u_{n}^{*}$ is the function from the nonsmooth (P.S.) condition, and $u^{*} \in \partial \varphi(u)$ such that

$$
\left\langle u_{n}^{\prime \prime}-u^{\prime \prime}, u_{n}-u\right\rangle \rightarrow 0
$$

as $n \rightarrow \infty$, so

$$
\begin{aligned}
\left\langle u_{n}^{*}-u^{*}, u_{n}-u\right\rangle= & -\int_{0}^{T}\left({ }_{0}^{c} D_{t}^{\alpha}\left(u_{n}(t)-u(t)\right),{ }_{t}^{c} D_{T}^{\alpha}\left(u_{n}(t)-u(t)\right)\right) d t \\
& +\int_{0}^{T}\left(q_{n}(t)-q(t), u_{n}(t)-u(t)\right) d t \\
\geq & |\cos (\pi \alpha)|\left\|u_{n}-u\right\|_{\alpha}^{2}+\int_{0}^{T}\left(q_{n}(t)-q(t), u_{n}(t)-u(t)\right) d t,
\end{aligned}
$$

where $q_{n}(t) \in \partial\left(-F\left(t, u_{n}(t)\right)\right)$ and $q(t) \in \partial(-F(t, u(t)))$.

By (3.4) and (3.5), it is easy to verify that $\left\|u_{n}-u\right\|_{\alpha} \rightarrow 0$ as $n \rightarrow \infty$, and hence that $u_{n} \rightarrow u$ in $E^{\alpha}$. Thus, $\left\{u_{n}\right\}$ admits a convergent subsequence.

In view of (B3), there exist two positive constants $M^{\prime}$ and $r_{2}$ such that

$$
F(t, x) \geq M^{\prime}|x|^{\mu}
$$

for a.e. $t \in[0, T]$ and $|x| \geq r_{2}$. It follows from (A) that

$$
F(t, x) \geq-C|x|^{\alpha+1}-C_{0}|x|-F(t, 0)
$$

for all $|x| \leq r_{2}$ and a.e. $t \in[0, T]$. Therefore, we obtain

$$
F(t, x) \geq M^{\prime}|x|^{\mu}-M^{\prime} r_{2}^{\mu}-C r_{2}^{\alpha+1}-C_{0} r_{2}-F(t, 0)
$$

for all $x \in \mathbb{R}^{N}$ and a.e. $t \in[0, T]$.

For any $u \in E^{\alpha}$ with $u \neq 0, \lambda>0$, we have

$$
\begin{aligned}
\varphi(\lambda u) & =-\frac{1}{2} \int_{0}^{T}\left({ }_{0}^{c} D_{t}^{\alpha} u(t),{ }_{t}^{c} D_{T}^{\alpha} u(t)\right) d t-\int_{0}^{T} F(t, u(t)) d t \\
& \leq \frac{\lambda^{2}}{2|\cos (\pi \alpha)|}\|u\|_{\alpha}^{2}-M^{\prime} \int_{0}^{T}|\lambda u(t)|^{\mu} d t+r_{3} T \\
& \leq \frac{\lambda^{2}}{2|\cos (\pi \alpha)|}\|u\|_{\alpha}^{2}-M^{\prime} \lambda^{\mu}\|u(t)\|_{L^{\mu}}^{\mu}+r_{3} T
\end{aligned}
$$

by (3.6), where $r_{3}$ is a positive constant. Then there exists a sufficiently large $\lambda_{0}$ such that $\varphi\left(\lambda_{0} u\right) \leq 0$.

By (B2), there exists $\epsilon \in(0,|\cos (\pi \alpha)|)$ and $\delta>0$ such that

$$
F(t, x) \leq(|\cos (\pi \alpha)|-\epsilon)\left(\Gamma^{2}(\alpha+1) / 2 T^{2 \alpha}\right)|x|^{2}
$$

for a.e. $t \in[0, T]$ and $|x| \leq \delta$. 
Let $\rho:=\frac{\Gamma(\alpha)(2 \alpha-1)^{1 / 2}}{T^{\alpha-1 / 2}} \delta$ and $\sigma=\epsilon \rho^{2} / 2>0$. Then it follows from $(2.2)$ that

$$
\|u\|_{\infty} \leq \frac{T^{\alpha-1 / 2}}{\Gamma(\alpha)((\alpha-1) / 2+1)^{1 / 2}}\|u\|_{\alpha}=\delta
$$

for all $u \in E^{\alpha}$ with $\|u\|_{\alpha}=\rho$. Therefore, we have

$$
\begin{aligned}
\varphi(u) & =-\frac{1}{2} \int_{0}^{T}\left({ }_{0}^{c} D_{t}^{\alpha} u(t),{ }_{t}^{c} D_{T}^{\alpha} u(t)\right) d t-\int_{0}^{T} F(t, u(t)) d t \\
& \geq \frac{|\cos (\pi \alpha)|}{2}\|u\|_{\alpha}^{2}-(|\cos (\pi \alpha)|-\epsilon) \frac{\Gamma^{2}(\alpha+1)}{2 T^{2 \alpha}} \int_{0}^{T}|u(t)|^{2} d t \\
& \geq \frac{|\cos (\pi \alpha)|}{2}\|u\|_{\alpha}^{2}-\frac{1}{2}(|\cos (\pi \alpha)|-\epsilon)\|u\|_{\alpha}^{2} \\
& =\frac{1}{2} \epsilon\|u\|_{\alpha}^{2} \\
& =\sigma
\end{aligned}
$$

for all $u \in E^{\alpha}$ with $\|u\|_{\alpha}=\rho$. This implies all the conditions in Lemma 2.6 are satisfied, so there exists a critical point $u_{0}$ for $\varphi$ and $\varphi\left(u_{0}\right) \geq \sigma$, and this completes the proof.

Theorem 3.2 Let $F$ satisfy (A), (B1), (B3) and the following conditions:

(B4) there exist $\mu^{\prime}>2$ and $Q^{\prime}>0$ such that

$$
\limsup _{|x| \rightarrow+\infty} \frac{F(t, x)}{|x|^{\mu^{\prime}}} \leq Q^{\prime}
$$

uniformly for a.e. $t \in[0, T]$;

(B5) $F(t, x)=F(t,-x)$ for $t \in[0, T]$ and all $x$ in $\mathbb{R}^{N}$.

Then system (1.1) has an infinite number of solutions $u_{k}$ on $E^{\alpha}$ for every positive integer $k$ such that $\left\|u_{k}\right\|_{\infty} \rightarrow+\infty$, as $k \rightarrow \infty$.

Proof The proof that the functional $\varphi$ satisfies the nonsmooth (P.S.) condition is the same as that of Theorem 3.1, so we omit it. We only need to verify other conditions in Lemma 2.7.

Since $E^{\alpha}$ is a separable and reflexive Banach space, there exist (see [38]) $\left\{e_{n}\right\}_{n=1}^{\infty} \subset E^{\alpha}$ and $\left\{f_{n}\right\}_{n=1}^{\infty} \subset\left(E^{\alpha}\right)^{*}$ such that

$$
\begin{aligned}
& f_{n}\left(e_{m}\right)=\delta_{n, m}= \begin{cases}1, & n=m, \\
0, & n \neq m,\end{cases} \\
& E^{\alpha}=\overline{\operatorname{span}\left\{e_{n}: n=1,2, \ldots\right\}} \text { and }\left(E^{\alpha}\right)^{*}=\overline{\operatorname{span}\left\{f_{n}: n=1,2, \ldots\right\}} W^{*} .
\end{aligned}
$$

For $k=1,2, \ldots$, denote

$$
X_{k}=\operatorname{span}\left\{e_{k}\right\}, \quad Y_{k}=\bigoplus_{j=1}^{k} X_{j}, \quad Z_{k}=\overline{\bigoplus_{j=k}^{\infty} X_{j}} .
$$


For any $u \in Y_{k}$, let

$$
\|u\|_{*}:=\left(\int_{0}^{T}|u(t)|^{\mu} d t\right)^{1 / \mu}
$$

and it is easy to verify that $\|\cdot\|_{*}$ defined by (3.9) is a norm of $Y_{k}$. Since all the norms of a finite dimensional normed space are equivalent, there exists a positive constant $C_{5}$ such that

$$
C_{1}\|u\| \leq\|u\| * \quad \text { for } u \in Y_{k}
$$

In view of (B3), there exist two positive constants $M_{1}$ and $C_{2}$ such that

$$
F(t, x) \geq M_{1}|x|^{\mu}
$$

for a.e. $t \in[0, T]$ and $|x| \geq C_{2}$. It follows from (3.10) and (3.11) that

$$
\begin{aligned}
\varphi(u)= & -\frac{1}{2} \int_{0}^{T}\left({ }_{0}^{c} D_{t}^{\alpha} u(t),{ }_{t}^{c} D_{T}^{\alpha} u(t)\right) d t-\int_{0}^{T} F(t, u(t)) d t \\
\leq & \frac{1}{2|\cos (\pi \alpha)|}\|u\|_{\alpha}^{2}-\int_{\Omega_{3}} F(t, u(t)) d t-\int_{\Omega_{4}} F(t, u(t)) d t \\
\leq & \frac{1}{2|\cos (\pi \alpha)|}\|u\|_{\alpha}^{2}-M_{1} \int_{\Omega_{3}}|u(t)|^{\mu} d t-\int_{\Omega_{4}} F(t, u(t)) d t \\
= & \frac{1}{2|\cos (\pi \alpha)|}\|u\|_{\alpha}^{2}-M_{1} \int_{0}^{T}|u(t)|^{\mu} d t \\
& +M_{1} \int_{\Omega_{4}}|u(t)|^{\mu^{\prime}} d t-\int_{\Omega_{4}} F(t, u(t)) d t \\
\leq & \frac{1}{2|\cos (\pi \alpha)|}\|u\|_{\alpha}^{2}-C_{1}^{\mu} M_{1}\|u\|^{\mu}+C_{3},
\end{aligned}
$$

where $\Omega_{3}:=\left\{t \in[0, T] ;|u(t)| \geq C_{2}\right\}, \Omega_{4}:=[0, T] \backslash \Omega_{3}$. Since $\mu>2$, there exists a positive constant $d_{k}$ such that

$$
\varphi(u) \leq 0 \quad \text { for all } u \in Y_{k} \text { and }\|u\| \geq d_{k} .
$$

For any $u \in Z_{k}$, let

$$
\|u\|_{\mu}:=\left(\int_{0}^{T}|u(t)|^{\mu^{\prime}} d t\right)^{1 / \mu^{\prime}} \quad \text { and } \quad \beta_{k}:=\sup _{u \in Z_{k},\|u\|=1}\|u\|_{\mu^{\prime}}
$$

then we conclude $\beta_{k} \rightarrow 0$ as $k \rightarrow \infty$. In fact, it is obvious that $\beta_{k} \geq \beta_{k+1}>0$, so $\beta_{k} \rightarrow \alpha \geq 0$ as $k \rightarrow \infty$. For every $k \in \mathbb{N}$, there exists $u_{k} \in Z_{k}$ such that

$$
\left\|u_{k}\right\|=1 \text { and }\left\|u_{k}\right\|_{\mu^{\prime}}>\beta_{k} / 2 \text {. }
$$

As $E^{\alpha}$ is reflexive, $\left\{u_{k}\right\}$ has a weakly convergent subsequence, still denoted by $\left\{u_{k}\right\}$, such that $u_{k} \rightarrow u$. We claim $u=0$. In fact, for any $f_{m} \in\left\{f_{n}: n=1,2, \ldots\right\}$, we have $f_{m}\left(u_{k}\right)=0$, when 
$k>m$, so

$$
f_{m}\left(u_{k}\right) \rightarrow 0, \quad \text { as } k \rightarrow \infty
$$

for any $f_{m} \in\left\{f_{n}: n=1,2, \ldots\right\}$, therefore $u=0$.

By Proposition 2.3, when $u_{n} \rightarrow 0$ in $E^{\alpha}$, then $u_{k} \rightarrow 0$ strongly in $C\left([0, T] ; \mathbb{R}^{N}\right)$. So we conclude $\alpha=0$ by (3.14). In view of (B4), there exist two positive constants $M_{2}$ and $C_{4}$ such that

$$
F(t, x) \leq M_{2}|x|^{\mu^{\prime}}
$$

for a.e. $t \in[0, T]$ and $|x| \geq C_{4}$. We conclude

$$
\begin{aligned}
\varphi(u)= & -\frac{1}{2} \int_{0}^{T}\left({ }_{0}^{c} D_{t}^{\alpha} u(t),{ }_{t}^{c} D_{T}^{\alpha} u(t)\right) d t-\int_{0}^{T} F(t, u(t)) d t \\
\geq & \frac{|\cos (\pi \alpha)|}{2}\|u\|_{\alpha}^{2}-\int_{\Omega_{5}} F(t, u(t)) d t-\int_{\Omega_{6}} F(t, u(t)) d t \\
\geq & \frac{|\cos (\pi \alpha)|}{2}\|u\|_{\alpha}^{2}-M_{2} \int_{0}^{T}|u(t)|^{\mu^{\prime}} d t \\
& +M_{2} \int_{\Omega_{6}}|u(t)|^{\mu^{\prime}} d t-\int_{\Omega_{6}} F(t, u(t)) d t \\
\geq & \frac{|\cos (\pi \alpha)|}{2}\|u\|_{\alpha}^{2}-M_{2} \beta_{k}^{\mu^{\prime}}\|u\|^{\mu^{\prime}}-C_{9},
\end{aligned}
$$

by (3.15), where $\Omega_{5}:=\left\{t \in[0, T] ;|u(t)| \geq C_{4}\right\}, \Omega_{6}:=[0, T] \backslash \Omega_{5}$.

Choosing $r_{k}=1 / \beta_{k}$, it is obvious that

$$
r_{k} \rightarrow \infty, \quad \text { as } k \rightarrow \infty
$$

then

$$
b_{k}:=\inf _{u \in Z_{k},\|u\|=r_{k}} \varphi(u) \rightarrow \infty, \quad \text { as } k \rightarrow \infty,
$$

that is, condition (A3) in Lemma 2.7 is satisfied. In view of (3.12), let $\rho_{k}:=\max \left\{d_{k}, r_{k}+1\right\}$, then

$$
a_{k}:=\max _{u \in Y_{k},\|u\|=\rho_{k}} \varphi(u) \leq 0
$$

and this shows condition (A2) in Lemma 2.7 is satisfied.

We have proved the functional $\varphi$ satisfies all the conditions of Lemma 2.7, then $\varphi$ has an unbounded sequence of critical values $c_{k}=\varphi\left(u_{k}\right)$ by Lemma 2.7; we only need to show $\left\|u_{k}\right\|_{\infty} \rightarrow \infty$ as $k \rightarrow \infty$.

In fact, since $u_{k}$ is a critical point of the functional $\varphi$, that is, $0 \in \partial \varphi\left(u_{k}\right)$, by Lemma 2.3 and Remark 2.7, we have

$$
-\int_{0}^{T}\left({ }_{0}^{c} D_{t}^{\alpha} u_{n}(t),{ }_{t}^{c} D_{T}^{\alpha} u_{n}(t)\right) d t+\int_{0}^{T}\left(u_{k}^{*}(t), u_{k}(t)\right) d t=0,
$$


where $u_{k}^{*}(t) \in \partial\left(-F\left(t, u_{k}(t)\right)\right)$. Hence, we have

$$
\begin{aligned}
c_{k} & =\varphi\left(u_{k}\right) \\
& =-\frac{1}{2} \int_{0}^{T}\left({ }_{0}^{c} D_{t}^{\alpha} u(t),{ }_{t}^{c} D_{T}^{\alpha} u(t)\right) d t-\int_{0}^{T} F\left(t, u_{k}(t)\right) d t \\
& =\frac{1}{2} \int_{0}^{T}\left(u_{k}^{*}(t), u_{k}(t)\right) d t-\int_{0}^{T} F\left(t, u_{k}(t)\right) d t \\
& \leq 2 T\left(C\left\|u_{k}\right\|_{\infty}^{\alpha+1}+C_{0}\left\|u_{k}\right\|_{\infty}\right)-\int_{0}^{T} F(t, 0) d t,
\end{aligned}
$$

since $c_{k} \rightarrow \infty$, it is obvious that

$$
\left\|u_{k}\right\|_{\infty} \rightarrow \infty \quad \text { as } k \rightarrow \infty
$$

by (3.14). This completes the proof of Theorem 3.2.

Theorem 3.3 Let $F(t, x)$ satisfy the condition $(A)$ with $v \in[0,1)$. Then $B V P(1.1)$ has at least one solution which minimizes $\varphi$ on $E^{\alpha}$.

Proof By (3.1), we obtain

$$
\begin{aligned}
\int_{0}^{T} & {[F(t, u(t))-F(t, 0)] d t+\int_{0}^{T} F(t, 0) d t } \\
= & \int_{0}^{T} \int_{0}^{1}(\partial F(t, s u(t)), u(t)) d s d t+\int_{0}^{T} F(t, 0) d t \\
\leq & \int_{0}^{T} \int_{0}^{1} f(t)|s u(t)|^{\alpha}|u(t)| d s d t \\
& +\int_{0}^{T} \int_{0}^{1} g(t)|u(t)| d s d t+\int_{0}^{T} F(t, 0) d t \\
\leq & \|u\|_{\infty}^{\nu+1} \int_{0}^{T} f(t) d t+\|u\|_{\infty} \int_{0}^{T} g(t) d t+\int_{0}^{T} F(t, 0) d t \\
\leq & C_{1}^{v+1}\|u\|_{\alpha}^{v+1} \int_{0}^{T} f(t) d t+C_{3}\|u\|_{\alpha}+C_{4},
\end{aligned}
$$

where $C_{1}$ is defined in (2.9), $C_{3}$ and $C_{4}$ are constants. Hence, we get

$$
\begin{aligned}
\varphi(u) & =-\int_{0}^{T} \frac{1}{2}\left({ }_{0}^{c} D_{t}^{\alpha} u(t),{ }_{t}^{c} D_{T}^{\alpha} u(t)\right) d t-\int_{0}^{T} F(t, u(t)) d t, \\
& \geq \frac{|\cos (\pi \alpha)|}{2}\|u\|_{\alpha}^{2}-C_{1}^{v+1}\|u\|_{\alpha}^{v+1} \int_{0}^{T} f(t) d t-C_{3}\|u\|_{\alpha}-C_{4} .
\end{aligned}
$$

If $0 \leq v<1$, we have

$$
\varphi(u) \rightarrow+\infty, \quad \text { as }\|u\|_{\alpha} \rightarrow \infty
$$

According to the same arguments in [36], $\varphi$ is weakly lower semi-continuous. By Lemma 2.5, the proof of Theorem 3.3 is completed. 


\section{Example}

In this section, we give two examples to illustrate our results.

Example 4.1 In BVP (1.1), let

$$
F(t, x)= \begin{cases}|x|^{3}, & |x| \leq 1 \\ |x|^{4}, & |x|>1\end{cases}
$$

It is easy to verify all the conditions in Theorem 3.2, so BVP (1.1) has infinitely many solutions $\left(u_{n}\right)$ on $E^{\alpha}$ and $\left\|u_{n}\right\|_{\infty} \rightarrow \infty$ as $n \rightarrow \infty$.

Example 4.2 In BVP (1.1), let $F(t, x)=|x|$. It is easy to verify all the conditions in Theorem 3.3, so BVP (1.1) has at least one solution which minimizes $\varphi$ on $E^{\alpha}$.

\section{Competing interests}

The authors declare that they have no competing interests.

\section{Authors' contributions}

All authors read and approved the final manuscript.

\section{Acknowledgement}

The authors thank the anonymous referees for valuable suggestions and useful hints from others.

Received: 7 May 2012 Accepted: 18 July 2012 Published: 31 July 2012

\section{References}

1. Kilbas, AA, Srivastava, HM, Trujillo, JJ: Theory and Applications of Fractional Differential Equations. North-Holland Mathematics Studies, vol. 204. Elsevier, Amsterdam (2006)

2. Miller, KS, Ross, B: An Introduction to the Fractional Calculus and Differential Equations. Wiley, New York (1993)

3. Podlubny, I: Fractional Differential Equations. Academic Press, San Diego (1999)

4. Samko, SG, Kilbas, AA, Marichev, Ol: Fractional Integral and Derivatives: Theory and Applications. Gordon \& Breach, Longhorne (1993)

5. Agarwal, RP, Benchohra, M, Hamani, S: A survey on existence results for boundary value problems of nonlinear fractional differential equations and inclusions. Acta Appl. Math. 109, 973-1033 (2010)

6. Lakshmikantham, V, Vatsala, AS: Basic theory of fractional differential equations. Nonlinear Anal. 69, $2677-2682$ (2008)

7. Vasundhara Devi, J, Lakshmikantham, V: Nonsmooth analysis and fractional differential equations. Nonlinear Anal. 70 4151-4157 (2009)

8. Bai, Z, Lü, H: Positive solutions for boundary value problem of nonlinear fractional differential equation. J. Math. Anal. Appl. 311, 495-505 (2005)

9. Agarwal, RP, O'Regan, D, Staněk, S: Positive solutions for Dirichlet problems of singular nonlinear fractional differential equations. J. Math. Anal. Appl. 371, 57-68 (2010)

10. Goodrich, CS: Existence of a positive solutions to a class of fractional differential equations. Appl. Math. Lett. 23, 1050-1055 (2010)

11. Kosmatov, N: A boundary value problem of fractional order at resonance. Electron. J. Differ. Equ. 135, 1-10 (2010)

12. Jiang, $W$ : The existence of solutions for boundary value problems of fractional differential equations at resonance. Nonlinear Anal. (2010). doi:10.1016/j.na.2010.11.005

13. Zhang, S: Existence of a solution for the fractional differential equation with nonlinear boundary conditions. Comput. Math. Appl. 61, 1202-1208 (2011)

14. Liang, S, Zhang, J: Positive solutions for boundary value problems of nonlinear fractional differential equation Nonlinear Anal. 71, 5545-5550 (2009)

15. Zhao, Y, Sun, S, Han, Z, Li, Q: The existence of multiple positive solutions for boundary value problems of nonlinear fractional differential equations. Commun. Nonlinear Sci. Numer. Simul. 16, 2086-2097 (2011)

16. Mawhin, J, Willem, M: Critical Point Theory and Hamiltonian Systems. Springer, New York (1989)

17. Rabinowitz, PH: Minimax Methods in Critical Point Theory with Applications to Differential Equations. CBMS, vol. 65 Am. Math. Soc., Providence (1986)

18. Ambreosetti, A, Rabinowitz, PH: Dual variational methods in critical point theory and applications. J. Funct. Anal. 14, 349-381 (1973)

19. Chang, K: Variational methods for non-differential functions and their applications to partial differential equations. J. Math. Anal. Appl. 80, 102-129 (1981)

20. Clarke, FH: Optimization and Nonsmooth Analysis. Classics in Appl. Math., vol. 5. SIAM, Philadelphia (1990)

21. Kourogenis, NC, Papageorgiou, NS: Nonsmooth critical point theory and nonlinear elliptic equations at resonance. J. Aust. Math. Soc. 69, 245-271 (2000) 
22. Halidias, N: Critical point theory for nonsmooth energy functionals and applications. Acta Math. Univ. Comen. 2, 147-155 (2002)

23. Kandilakis, D, Kourogenis, NC, Papageorgiou, NS: Two nontrivial critical points for nonsmooth functionals via local linking and applications. J. Glob. Optim. 34, 219-244 (2006)

24. Liu, J, Guo, Y: Critical point theory for nonsmooth functions. Nonlinear Anal. 66, 2731-2741 (2007)

25. Marano, S, Motreanu, D: Infinitely many critical points of non-differentiable functions and applications to a Neumann-type problem involving the $p$-Laplacian. J. Differ. Equ. 182, 108-120 (2002)

26. Marano, S, Motreanu, D: On a three critical points theorem for non differentiable functions and applications to nonlinear boundary value problems. Nonlinear Anal. 48, 37-52 (2002)

27. Dai, G: Nonsmooth version of Fountain theorem and its application to a Dirichlet-type differential inclusion problem. Nonlinear Anal. 72, 1454-1461 (2010)

28. Filippakisa, M, Gasińskib, L, Papageorgiou, NS: Periodic problems with asymmetric nonlinearities and nonsmooth potentials. Nonlinear Anal. 58, 683-702 (2004)

29. Filippakis, ME: Existence and multiplicity results for nonlinear nonautonomous second-order systems. Nonlinear Anal. 68, 1611-1626 (2008)

30. Hu, S, Papageorgiou, NS: Positive solutions and multiple solutions for periodic problems driven by scalar $p$-Laplacian. Math. Nachr. 279, 1321-1334 (2006)

31. Kristly, A: Infinitely many solutions for a differential inclusion problem in $\mathbb{R}^{N}$. J. Differ. Equ. 220, $511-530$ (2006)

32. Gasinski, L, Papageorgiou, NS: Nonsmooth Critical Point Theory and Nonlinear Boundary Value Problems. Chapman \& Hall/CRC Press, Boca Raton (2005)

33. Ouahab, A: Some results for fractional boundary value problem of differential inclusions. Nonlinear Anal. 69, 3877-3896 (2008)

34. Chang, Y-K, Nieto, JJ: Some new existence results for fractional differential inclusions with boundary conditions Math. Comput. Model. 49, 605-609 (2009)

35. Wang, J, Zhou, Y: Existence and controllability results for fractional semilinear differential inclusions. Nonlinear Anal., Real World Appl. 12, 3642-3653 (2012)

36. Jiao, F, Zhou, Y: Existence of solutions for a class of fractional boundary value problem via critical point theory. Comput. Math. Appl. 62, 1181-1199 (2011)

37. Bartsch, T: Infinitely many solutions of a symmetric Dirichlet problem. Nonlinear Anal. 68, 1205-1216 (1993)

38. Fan, $X L, H a n, X Y$ : Existence and multiplicity of solutions for $p(x)$-Laplacian equations in $\mathbb{R}^{N}$. Nonlinear Anal. 59, $173-188(2004)$

doi:10.1186/1687-2770-2012-82

Cite this article as: Zhang and Gong: Existence and multiplicity results for a class of fractional differential inclusions

with boundary conditions. Boundary Value Problems 2012 2012:82.

\section{Submit your manuscript to a SpringerOpen ${ }^{\circ}$ journal and benefit from:}

- Convenient online submission

- Rigorous peer review

- Immediate publication on acceptance

Open access: articles freely available online

High visibility within the field

- Retaining the copyright to your article 